\title{
Matemáticas: Patrones de Interacción Discursivos en un Curso de Enseñanza Media
}

\author{
Hermes Nolasco-Hesiquio ${ }^{(1)^{\star}}$, Guadalupe Cabañas-Sánchez ${ }^{(1)}$, Osvaldo Rojas ${ }^{(2)}$ y José M. Sigarreta ${ }^{(1)}$ \\ (1) Programa de Maestría en Matemática Educativa, Facultad de Matemáticas, Universidad Autónoma de \\ Guerrero, Av. Lázaro Cárdenas S/N, Ciudad Universitaria, Chilpancingo, Gro. México. \\ (e-mail:nolascohh@hotmail.com; gcabanas.sanchez@gmail.com; josemariasigarretaalmira@yahoo.es) \\ (2) Programa de Maestría y Doctorado en Educación Matemática, Universidad Antonio Nariño, Carrera 38 \\ No.58A-77, Nicolas de Federman, Bogotá, Colombia (e-mail: orojasv69@uan.edu.co) \\ *autor a quien debe ser dirigida la correspondencia.
}

Recibido Mar. 31, 2016; Aceptado May. 23, 2016; Versión final Jul. 18, 2016, Publicado Dic. 2016

\section{Resumen}

Se presenta un estudio sobre el discurso del profesor en el aula de matemáticas, cuando se pretende enseñar conceptos y procesos matemáticos ligados a la noción de semejanza. Se pretende identificar los patrones de interacción que regulan las formas de actuación que han de ser válidas para la construcción de consensos en el aula. Se adopta como marco teórico las estrategias y los principios desarrollados por la perspectiva interaccionista y el análisis del discurso. Consideraremos un modelo de investigación cualitativa, basado en el método etnográfico. Los datos del estudio proceden de sesiones de matemáticas en un aula de Enseñanza Media (16-18 años). Los resultados muestran que por medio del adecuado discurso se facilita la identificación de patrones de interacción entre el profesor y sus estudiantes.

Palabras clave: patrones de interacción, análisis del discurso, interaccionismo, semejanza, método etnográfico

\section{Mathematics: Discursive Interaction Patterns in a High School Course}

\begin{abstract}
A study on the teacher's speech in the mathematics classroom, when trying to teach concepts and mathematical processes linked to the notion of similarity is presented. The main objective is to identify the interaction patterns that rule behaviors that must be valid to create consensus in the classroom. The theoretical framework adopted included the principles and strategies developed by the interactionism perspective and discourse analysis. A qualitative research model based in the ethnographic method was considered. Data for the study were taken from the Math lessons in a high school course (ages 16-18). The results demonstrate that appropriate speech simplifies the identification of interaction patterns between the professor and the students.
\end{abstract}

Keywords: interaction patters, discourse analysis, interactionism, similarity, ethnographic method 


\section{INTRODUCCIÓN}

El trabajo del profesor de matemáticas se realiza dentro de un espacio social específico: la institución escolar. Parecería obvia esta afirmación, pero a pesar de ello, existe poca reflexión sobre su contexto cotidiano de trabajo (Ponte y Chapman, 2016). Esta carencia es especialmente palpable en las instituciones de formación y de actualización docente, en las cuales se observa una gran desvinculación entre los contenidos de formación y la realidad escolar que enfrentan los maestros. Es imposible inferir esta problemática asociada con la institución escolar a partir, de manera directa, de la evaluación actual. Consideramos que la reconstrucción de esa lógica requiere de un análisis de las prácticas educativas, que explicite lo que sucede cotidianamente en la escuela.

La importancia del estudio de la semejanza en la escuela Media Universitaria, en lo fundamental, está dada por las dificultades metodológicas asociadas con su proceso de enseñanza-aprendizaje, además de su potencialidad como articulador de nociones matemáticas generales, como la proporcionalidad y el Teorema de Thales. En esa misma dirección, resulta atinado plantear, que existen escasos estudios sobre la adquisición de la noción semejanza por los estudiantes; por ende, poco es lo que sabemos sobre la forma en que los docentes enfrentan su tratamiento metodológico y qué adaptaciones se necesitan realizar a los programas vigentes, en los cuales el contenido no sólo se desdibujó, sino también se borraron sus relaciones con otros temas matemáticos.

El hecho de analizar la situación concreta de un contenido programático, en la escuela, puede verse influido por las normas lingüísticas implícitas en el discurso del profesores o bien, por las relaciones de poder y autoridad que existen entre maestros y alumnos. De alguna manera, estas múltiples relaciones del proceso escolar impactan significativamente en las oportunidades que tienen los alumnos de aprender. De allí, la importancia de estudiar el discurso del profesor a partir de la situación sociocultural en que se desarrolla la actividad. En ese sentido, pensamos que un primer paso para la reflexión y el análisis del trabajo docente puede partir de un estudio dirigido al profesor y, en particular, al análisis y a la interpretación de las interacciones discursivas que mantienen los profesores de matemáticas con sus estudiantes. Esta investigación se centra en un contenido específico: la enseñanza-aprendizaje de la semejanza en la Enseñanza Media Universitaria.

En los últimos años, se ha incrementado notablemente el número de investigaciones que se han ocupado de comprender la práctica del profesor de matemáticas. Algunos trabajos están orientados a identificar la influencia de los diferentes dominios del conocimiento del profesor en relación con la práctica social (Gavilán et al., 2007; Godino, 2009; Barboza y Zapata, 2013). También desde la teoría de situaciones didácticas y la transposición didáctica algunos investigadores analizan las prácticas del profesor en clases ordinarias (Chevallard, 2002; Perrin Glorian y Hersant, 2003; Herbst et al., 2011; Brousseau et al., 2014). Diversos estudios desde la perspectiva interaccionista (Bauersfeld, 1995; Knipping, 2008; Krummheuer, 2011) han definido formatos o patrones de interacción del profesor con sus estudiantes, en el que por medio del discurso los significados matemáticos son construidos interactivamente en el salón de clase.

En este ámbito, algunos autores con distintas orientaciones teóricas coinciden al reconocer que el discurso que se produce en el aula y los patrones de interacción que se presentan en ese espacio pueden influir en las oportunidades que tengan los alumnos para aprender (Lemke, 1997; Mercer, 2001; Contreras, 2016). Se podría decir que estas se presentan en función de actos de apertura o de cierre respecto a la participación significativa del alumno durante el proceso de construcción del conocimiento en el aula. Si los alumnos no participan como interlocutores, como compañeros en el diálogo, si no confrontan puntos de vista y emiten juicios sobre la validez de lo propuesto en clase, no podrán hacer suyos los argumentos a los que se enfrentan.

En diferentes países se han hecho investigaciones sobre la interacción que existe entre maestro y alumnos en clases de matemáticas en situaciones cotidianas (Voigt, 1995; Cobb y Bauersfeld, 1995; Pimm, 1999; Gresalfi y Cobb, 2011; Planas, 2014). En México, algunos de los aspectos explorados en relación con la interacción discursiva se encuentran en los trabajos de Candela (1999), quien presenta una discusión amplia sobre los mismos, muestra cómo los alumnos no siempre asumen un papel activo en el aula, es decir, reclaman su derecho a justificar razones acerca de lo expresado por el profesor. El alumno, entonces, no debe limitarse a responder, sino también a formular preguntas para, de alguna forma, incidir en la interacción, puesto que los maestros tienen que reaccionar a sus contribuciones.

Uno de los problemas que enfrentan día a día los profesores de Enseñanza Media es la diversidad de actividades que se ponen en marcha para trabajar los contenidos escolares. La literatura revisada indica que el estudio de las formas discursivas, como se ha mencionado, ofrece algunas respuestas sobre cómo el discurso revela turnos de interacción que se encaminan a aportar información específica sobre el proceso 
de enseñanza-aprendizaje en el contexto escolar. Tal situación, nos permitió delimitar y centrar un aspecto particular del problema de investigación a través de la siguiente pregunta: ¿Cuáles son los patrones de interacción discursivos en la clase de Geometría? En específico, nos planteamos como objetivo, identificar los patrones de interacción que regulan las formas de actuación que han de ser válidas para la construcción de consensos en el aula. Poniendo énfasis en la actuación o manifestación (lingüística o no) del profesor al resolver problemas de semejanza, y al momento de argumentar, validar y comunicar la solución a sus alumnos.

\section{MATERIALES Y METODOS}

Este trabajo toma como marco teórico las estrategias y los principios desarrollado por la perspectiva interaccionista y análisis del discurso. Somos conscientes de que estas perspectivas se han desarrollado en contextos diferentes. Por lo tanto, no pretendemos profundizar en sus aportaciones en los campos científicos en donde fueron desarrolladas, ni elaborar una convergencia forzada de ideas y principios. Sin embargo, en nuestro proceso de estudio y profundización teórica, y en busca de soluciones, pretendemos desde perspectivas preestablecidas construir una base para el análisis de la interacción educativa coherente y capaz de describir y sintetizar el discurso del profesor en aquellos elementos que son objeto de estudio. Hemos optado por adentrarnos en el estudio del discurso en el aula a partir de las interacciones discursivas que tienen lugar entre profesores y alumnos cuando se enseña la noción de semejanza.

De acuerdo con la síntesis que realizan Sierpinska y Lerman (1996) del programa interaccionista, aplicado a la Matemática Educativa, el interaccionismo es una de las aproximaciones a la investigación sobre el desarrollo intelectual que promueve una visión sociocultural sobre las fuentes y el crecimiento del conocimiento. En este sentido, la aproximación interaccionista enfatiza como objeto de estudio en las interacciones entre individuos dentro de un mismo medio sociocultural. Uno de los supuestos adoptados en esta investigación, se orientan a estudiar la noción de negociación de significados en la interacción, que en síntesis consiste en la construcción interactiva de la intersubjetividad de los participantes y no tanto la situación sociocultural donde se dan los acontecimientos. Efectivamente, los participantes tienen que negociar el significado con el fin de llegar a un significado compartido. Por medio de la negociación del significado, las concepciones individuales se hacen compatibles a todos, de modo que los individuos interactúan, como si adscribieran el mismo significado a los objetos estudiados.

Como afirman Sierpinska y Lerman (1996), el fin de la mayor parte de la investigación del programa interaccionista en la Educación Matemática es lograr una mejor comprensión de los fenómenos de enseñanza y aprendizaje, tal y como ocurre en los contextos escolares ordinarios. Como podemos ver, esta perspectiva subraya la importancia de la «constitución interactiva» del significado en la clase y convierte un objeto de investigación las relaciones entre las características sociales de los procesos de interacción, así como las existentes entre el pensamiento del profesor y de los estudiantes.

En la clase de matemáticas, la construcción individual de significados tiene lugar en la interacción con la cultura de la clase, mientras que al mismo tiempo contribuye a la constitución de esta cultura (Cobb y Bauersfeld, 1995). Como podemos ver esta perspectiva subraya la importancia de la «constitución interactiva» del significado en las aulas y asocia un objeto de investigación con las relaciones entre las características sociales de los procesos de interacción, el pensamiento del profesor y de los estudiantes. Para el interaccionismo, la construcción individual de los significados en la clase de matemáticas tiene lugar en interacción con la cultura predominante de la clase (Cobb y Bauersfeld, 1995). De esta manera, el aprendizaje describe un proceso personal de formación, un proceso de adaptación interactiva a una cultura a través de la participación activa en dicha cultura.

Desde esta perspectiva interaccionista, las diversas construcciones subjetivas del significado y la necesidad de llegar a adaptaciones viables de «significados y regularidades compartidas», requiere oportunidades para las discusiones y para la negociación de los significados. La negociación de significados matemáticos en clase implica que cada uno de los participantes, profesor y alumnos, se formen sus propios significados visibles en el proceso. Desde esta perspectiva, el significado matemático no es tomado como existente, independientemente de los individuos que actúan y de su interacción, sino que se ve generado en el curso de la interacción social. En esta interacción tiene un papel preponderante el discurso, que tiene que ver con compartir significados. En particular, para Sfard (2007) el discurso matemático es aquel que utiliza palabras relacionadas con cantidades o figuras o símbolos creados especialmente para facilitar esta forma particular de comunicación.

Para la identificación de patrones de interacción, en particular, seguimos los estudios realizados por Voigt (1995) que considera como regularidades que son interactivamente constituidos por el profesor y los estudiantes. Son una consecuencia de la tendencia natural de las interacciones humanas más predecibles, 
menos arriesgadas en su organización y evolución. Dichas regularidades nos permitirán la identificación de patrones de interacción al desarrollar en una clase de Geometría la noción de Semejanza.

En el presente trabajo de investigación se ha optado por un enfoque etnográfico. Dicha perspectiva permite reconstruir cualitativa y descriptivamente lo que se dice y hace en el aula (Erickson, 1986). Es decir, estamos interesados en el punto de vista y en la perspectiva de los participantes, pues creemos que es posible comprender la intrincada red de relaciones y de acontecimientos que tienen lugar en una realidad particular. Sólo así es posible entender la significación que profesor y alumnos le otorgan a sus acciones. Para ello, es necesario, como ya se ha argumentado, analizar las acciones discursivas en su entorno natural de producción considerando que el habla se origina secuencialmente en un contexto discursivo.

La etnografía como enfoque de investigación observacional, participativo e interpretativo (Bertely, 2010) es un proceso de investigación que refiere a una descripción. De acuerdo con Geertz (1987), hacer etnografía no es sólo cuestión de método, se define por una clase de esfuerzo intelectual mediante el cual se hace una descripción profunda de la realidad. En esta descripción, compleja, se trata de descubrir una jerarquía estratificada de estructuras significativas bajo cuyas formas y acciones son producidas, percibidas e interpretadas. Asimismo, la etnográfica basada en la observación intensiva, constituye una alternativa metodológica para el estudio que pretendemos realizar. A través de ellas -técnica de observación y análisis cualitativo de datos- se puede favorecer la comprensión y sistematización de los procesos de aprendizaje que aparecen en el aula.

\section{Contexto y participantes}

El Sistema Educativo Mexicano se compone de tres niveles: Educación Básica, que incluye preescolar, primaria y secundaria; Educación Media Superior, que comprende el bachillerato, y Educación Superior. En el caso de la Educación Media Superior, ésta se distingue por su heterogeneidad institucional, con diversas modalidades y orientaciones como: Bachillerato Tecnológico, Bachillerato General y la Enseñanza Media ofertadas por las Universidades Públicas Nacionales y Estatales. En este contexto, la parte empírica del estudio se ha llevado a cabo en tres aulas de matemáticas del tercer semestre de Enseñanza Media (16-18 años) ubicadas en la Ciudad de Acapulco, México. En cada aula, se videograbaron sesiones de 50 minutos similares a las que cotidianamemte se desarrollan en clase. Las sesiones observadas pertenecen al contenido temático "semejanza" y forma parte del curso de Geometría.

Los participantes fueron profesores universitarios que laboran en preparatorias que ofrecen Enseñanza Media en la Universidad Autónoma de Guerrero. Los profesores que participaron lo hicieron de manera voluntaria y consintieron la intromisión en sus tareas docentes (observación de clases, grabación de video y audio, etc.). Los alumnos participaron a petición de su profesor. La elección de los profesores y de los alumnos que fueron grabados en video no se realizó bajo un criterio específico, sino que simplemente se tuvo en cuenta su valiosa disponibilidad para colaborar y la posibilidad de acceso al salón durante sus clases.

\section{RESULTADOS Y DISCUSIÓN}

En este apartado se caracterizarán los patrones de interacción discursivos manifestados en el aula, y para ejemplificar algunos de ellos se tomará la transcripción de algunas prácticas escolares realizadas por los profesores:

\section{P1. Los contenidos primero se explican y después se aplican a ejercicios concretos}

En el siguiente episodio, el profesor considera que los contenidos primero se explican y posteriormente se aplican a ejercicios concretos (ver tabla 1). En las clases del profesor la introducción de un objeto a conocer viene siempre seguida de momentos destinados a la práctica. Sin embargo, aquí una cuestión la hace singular: se hace hincapié en que la ejercitación es importante para reafirmar lo explicado en la clase: [387388] "Bien, si ha quedado claro, vamos a desarrollar un ejercicio, aplicando todo lo que hemos analizado, para poder reafirmar lo que acabamos de analizar como semejanza de triángulos...". En este caso, son temas que supuestamente los alumnos ya saben, esto es ejercitar el contenido que ya se ha visto.

En las intenciones del profesor se aprecia que los alumnos deben involucrar la incógnita " $x$ " y encontrar alguna relación entre el valor buscado y el criterio de semejanza. La importancia que para él tiene la visualización del dibujo significa la semejanza de figuras y en el paso a la expresión numérica se refleja la presentación del contenido matemático como se infiere en el siguiente segmento: [397-398] "Escriban: "Número uno. Aplicando los criterios de semejanza y tomando en cuenta que los ángulos son iguales y los lados son proporcionales. Encuentra el valor de " $x$ " en la siguiente figura..." 
Tabla 1. Los contenidos primero se explican y después se aplican a ejercicios concretos.

\begin{tabular}{|c|c|}
\hline $\begin{array}{l}387 \\
388 \\
389\end{array}$ & $\begin{array}{l}\text { Maestro: Bien, si ha quedado claro, vamos a desarrollar un ejercicio, aplicando todo lo que hemos } \\
\text { analizado, para poder reafirmar lo que acabamos de analizar como semejanza de triángulos. Vamos a } \\
\text { empezar. }\end{array}$ \\
\hline 390 & Alumnos: No. \\
\hline 391 & Maestro: ¿Ya terminaron? \\
\hline 392 & Alumna: No. \\
\hline 393 & Maestro: Bueno, vamos a aplicar lo que hemos analizado con respecto a la semejanza de triángulos. Los \\
\hline 394 & criterios, el uno, el dos y el tres nos van a servir para dar respuestas al siguiente ejercicio. Anoten por ahí \\
\hline 395 & "ejercicio". \\
\hline 396 & Alumno: Espéreme. \\
\hline 397 & Maestro: Escriban: Número uno. Aplicando los criterios de semejanza y tomando en cuenta que los ángulos \\
\hline 398 & uentra el valor de " $x$ " en la siguiente figura. Ten presente \\
\hline 399 & nde a las medidas (traza un triángulo-rectángulo en el \\
\hline 400 & pizarrón). \\
\hline 401 & \\
\hline 402 & A \\
\hline 403 & \\
\hline 404 & \\
\hline 405 & $\mathrm{x}$ \\
\hline 406 & $\underline{\mu}$ \\
\hline 407 & \\
\hline 408 & $\rightarrow \mathrm{c}$ \\
\hline 409 & $48^{B^{\prime}}$ \\
\hline $\begin{array}{l}410 \\
411\end{array}$ & \\
\hline $\begin{array}{l}411 \\
412\end{array}$ & Ahí tenemos la figura, a la cual vamos a dar respuesta, encontrando el valor de la variable " $x$ ", vamos a \\
\hline 413 & buscar el valor de " $x$ ", tomando en cuenta los criterios de semejanza que acabamos de analizar. Aquí se \\
\hline 414 & dice que el lado $A B$ es paralelo al lado $A^{\prime} B^{\prime}$ (señala en la figura) y además, dice que el lado $B C$ es igual a \\
\hline 415 & 48 y que $B$ 'C es igual a 3 . Ahora, vamos a encontrar cuánto vale " $x$ ", considerando los criterios de \\
\hline 416 & semejanza. ¿Cómo se les ocurre pensar en una posible solución?, ¿cómo pudiéramos analizar? (No hay \\
\hline 417 & respuesta de parte de los alumnos). ¿Qué diríamos?, ¿podríamos considerar que estos ángulos son \\
\hline 418 & iguales? (marca los dos ángulos rectos $B$ y $B^{\prime}$ del triángulo). Entonces diríamos: Si $A B=A^{\prime} B^{\prime}, \measuredangle B=\measuredangle B^{\prime}$, \\
\hline 419 & también podríamos afirmar que el ángulo $C$ es igual al ángulo $C^{\prime}$ porque corresponde a la misma figura, 0 \\
\hline 420 & sea, coinciden ¿verdad?, ¿si se dan cuenta? \\
\hline 421 & Alumnos: Pero, ¿ ¿de dónde sale el ángulo C’? \\
\hline 422 & Maestro: Si nosotros separamos la figura, ya que el ángulo $C$ \\
\hline
\end{tabular}

Para Mercer (2001) las preguntas del profesor se presentan como una técnica dominante para iniciar, extender y controlar la conversación en la clase. Por ejemplo, en el episodio de la tabla 1, el profesor plantea una serie de preguntas para extender la actividad [416] "¿Cómo se les ocurre pensar en una posible solución?, ¿cómo pudiéramos analizar?..” y, al no encontrar respuesta por parte de los alumnos, aporta información adicional al señalar la igualdad de los ángulos de los dos triángulos que se encuentran en el modo de configuraciones de Thales y la idea de transformar una figura en otra está ausente [418-420] "Entonces diríamos: $\mathrm{Si} A B=A^{\prime} B^{\prime}, \measuredangle B=\measuredangle B^{\prime}$ ', también podríamos afirmar que el ángulo $C$ es igual al ángulo $C^{\prime}$ porque corresponde a la misma figura...". Podemos dar cuenta en este episodio, que la parte procedimental juega un papel más importante que lo conceptual, el docente supone que el dominio de los procedimientos se logra a través de la práctica y con ello se asegura la comprensión de los principios que sustentan lo conceptual.

\section{P2. La explicación: una estrategia discursiva en la clase}

En las clases de matemáticas observadas, encontramos circunstancias donde aparece la explicación bajo las siguientes formas: las del profesor a los alumnos, las de los alumnos al profesor y las de un alumno a sus compañeros. La explicación se establece con reiteración bajo el despliegue de las siguientes estrategias: exposición oral del maestro, interrogatorio a los alumnos y explicación a través de un ejemplo. La exposición oral tiene pretensiones explicativas que, alternadas con el planteamiento de ejercicios, suponen la reafirmación de lo explicado en clase. En relación a esta última reflexión, Balacheff (1982) indica que "la explicación es un discurso tendiente a hacer inteligible el carácter de verdad de una proposición o un resultado". En este sentido, se puede decir que la explicación es uno de los medios que emplea el profesor para lograr la comprensión de un contenido. Por ejemplo, en la tabla 2 observaremos las explicaciones del profesor al hacer la introducción de la clase; el nuevo objeto a saber bajo la forma de definición: 
Tabla 2. La explicación: una estrategia discursiva en la clase

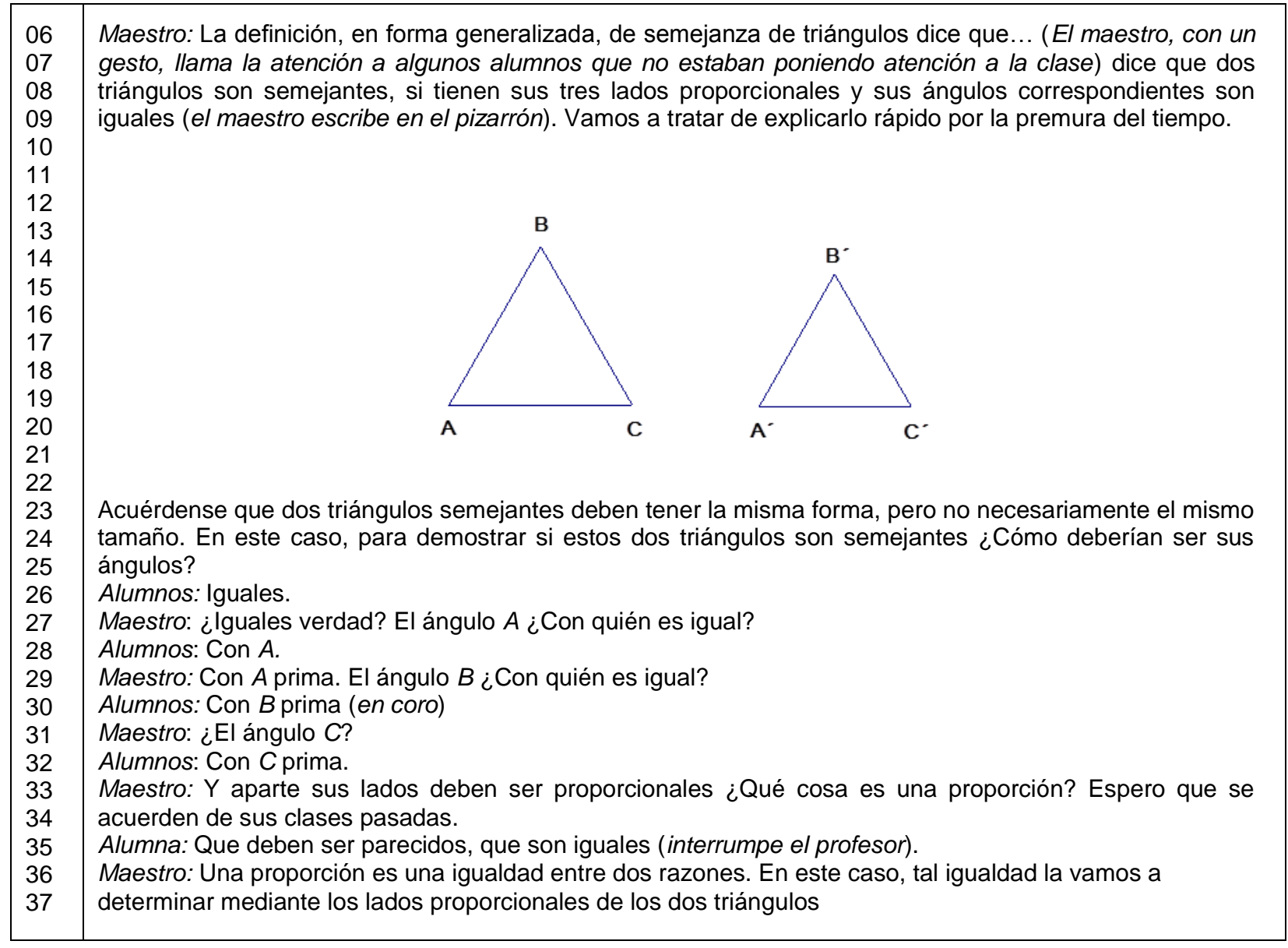

La idea de semejanza es introducida por una explicación, previa a la definición, que el alumno debe completar y en la que el profesor añade lo que considera faltante como para establecer la definición o el enunciado. Una estrategia dominante en las clases del profesor es la interrogación a los alumnos en su explicación oral: ([24-25] “¿Cómo deberían ser sus ángulos?”, [33] “¿Qué cosa es una proporción?”). Podemos afirmar que estas preguntas pueden estar enfocadas a la obtención de pistas o indicios sobre el estado y el avance del grupo en general, ya sea sobre los conocimientos previos o sobre los contenidos trabajados; o intentan incorporar los intereses, las experiencias y las ideas de los alumnos con el fin de orientar la discusión matemática. Para Si bien, al hacer uso de su lugar privilegiado en la relación, el profesor está en posibilidades de juzgar sin ofrecer derecho de réplica, así como de promover la ejecución de otros mecanismos para saber la veracidad de la actividad matemática de los alumnos.

\section{P3. Para que los conocimientos sean válidos, deben demostrarse}

En el episodio de la tabla 3, el profesor da inicio con una pregunta abierta para introducir al tema "semejanza" ([16] “¿Cómo logramos conocer el valor de "x" de este punto a este otro?", ([25-26] “¿Jonathan ¿todavía no?, ¿y el equipo de la esquina?") sin encontrar respuestas. Posteriormente, un alumno responde a los requerimientos y exigencias del profesor; hace uso de la cuarta proporcional sin reflexionar sobre la proporcionalidad de los lados de los triángulos. Por su parte, el profesor evalúa positivamente la explicación del alumno, ([40] "iAh! correcto, muy bien”) y valida las respuestas por consenso a través de la respuesta coreada del alumnado [44]. Lo anterior asume que una respuesta a coro equivale a la participación de toda o de casi toda la clase. En estos casos, el profesor generalmente acepta la respuesta con una afirmación y se dirige a algún alumno en especial y le solicita demostraciones y explicaciones concretas ([46] "Bueno, pero ¿cómo podemos demostrar que efectivamente es el resultado correcto?”). Y continúa instando al alumnado con insistencia: ([47-48] “... ¿pasarías a demostrarnos por qué el resultado es correcto?, ¿no consideramos que ese resultado es correcto?") Los alumnos responden nuevamente con una afirmación ([49] "sí maestro, sí es correcto"). El profesor intenta que los alumnos den el porqué de su declaración sin tener éxito y, finalmente, a través de un discurso retórico evalúa la actividad [50-52]. 
Tabla 3. Para que los conocimientos sean válidos, deben demostrarse

01

02

03

04

05

06

07

08

09

10

11

12

13

14

15

16

17

18

19

20

21

22

23

24

El profesor dibuja en el pizarrón un triángulo de la siguiente manera:

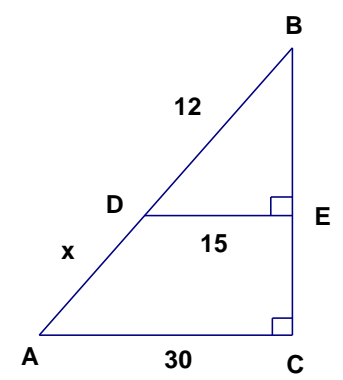

Maestro: ¿Cómo logramos conocer el valor de " $x$ " de este punto a este otro? (señala el segmento $A D$ del triángulo $A B C$ ). Necesitamos conocer este segmento. Les di los elementos necesarios la última sesión, la información la tienen en las hojas de trabajo, para que puedan resolverlo; de lo contrario, lo haremos entre todos. Tienen tres minutos para resolver el cuestionamiento de manera individual, en caso de que no lo logren pueden consultarlo con su compañero de butaca. Se le firmará a los primeros diez alumnos que encuentren el valor de " $x$ ".

El triángulo tiene medidas, no vayas a estar equivocado (le comenta a un alumno que ya resolvió el problema).

Alumno: Sí.

Maestro: Transcribe lo que hiciste al pizarrón (el alumno hace lo que se le ha indicado). Jonathan ¿todavía no?, ¿y el equipo de la esquina? (mientras, el alumno que pasa al pizarrón escribe:

$$
\begin{aligned}
& \frac{15}{12}=\frac{30}{x} \\
& x=\frac{(12)(30)}{15} \\
& x=24
\end{aligned}
$$

Maestro: Bien, ¿por qué no explicas a tus compañeros cómo hiciste para resolverlo? Por favor, guarde silencio quien venga llegando porque Moisés nos dirá cómo hizo para obtener el valor de " $x$ ".

Alumno: Yo tomé los ejemplos que nos había dado y que se parecen mucho

a éste, me di cuenta que dos lados se multiplican y uno se divide.

Maestro: ¡Ah! correcto, muy bien. Se puede decir que él obtuvo el valor de " $x$ " a través de un ejemplo anterior. Sin embargo, quisiera que fuéramos a algo más profundo, que comprendiéramos este ejemplo, así como los anteriores. Estamos hablando de proporciones y de magnitudes ¿no?, ¿estamos bien?, observamos que el resultado es correcto ¿no compañeros?

Alumnos: Sí (en coro).

Maestro: Bueno, pero ¿cómo podemos demostrar que efectivamente es el resultado correcto? Pasa compañero (se dirige a un alumno en específico), ¿pasarías a demostrarnos por qué el resultado es correcto? o ¿no consideramos que ese resultado es correcto? Porque se cumplirá con la igualdad de las razones.

Alumnos: Sí maestro, sí es correcto.

Maestro: A ver, alguien más que lo diga, a ver aquí (se dirige a Moisés y le indica que pase a explicar al frente). ¿Por qué dividimos 15 entre 12 y 30 entre 24? ¿Y qué nos da? Nos da la igualdad ¿verdad?, muy bien. ¿Quién trae el borrador? o ¿dónde está el borrador del grupo?...

El planteamiento de preguntas abiertas es un rasgo peculiar de muchas de las actividades que se llevan a cabo en las clases de matemáticas. El profesor inicia con una pregunta y luego sigue una serie de instrucciones para realizar la actividad [16-21]. A este tipo de preguntas le llamamos preguntas de continuidad puesto que son enunciados interrogativos generalmente breves que tienen la función de asegurar una continuidad del discurso y apelar la atención de los interlocutores. En este episodio, el profesor se aproxima al concepto de semejanza dentro de la relación intrafigural, pues la idea de transformar una figura en otra está ausente, considerando el aspecto de proyección (Ver tabla 3, líneas 215). Aunque el profesor no hace explícito que se trata de una configuración de Thales, se dirige directamente al modo de representación gráfico por medio de la identificación de datos numéricos/algebraicos con los correspondientes segmentos de la configuración y solicita el cálculo del valor numérico. En la tabla 4 recogemos las interacciones discursivas entre el profesor y sus estudiantes, identificadas en el análisis del episodio de la tabla 3. 
Tabla 4. Interacciones discursivas entre el profesor y sus estudiantes

\begin{tabular}{|l|l|}
\hline Discurso del profesor & Discurso del estudiante \\
\hline Plantea preguntas abiertas & Responde a los requerimientos y exigencias del profesor \\
\hline Demanda explicaciones & Provee justificaciones \\
\hline Valida las respuestas por consenso & Respuesta en coro \\
\hline Solicita demostraciones & Reafirman con un "sí" \\
\hline Utiliza un discurso retórico y evalúa la actividad & Discurso del estudiante \\
\hline
\end{tabular}

Los fenómenos matemáticos del discurso refieren aspectos que indican el desarrollo o el logro de una comprensión matemática. El intento del alumnado por justificar su asentimiento no fue el más idóneo como demostración en el contexto de este salón de clase. Una demostración formal requeriría de una cadena de razonamientos lógicos con el uso de definiciones, teoremas y axiomas. En este caso el alumno, apoyándose en los ejemplos dados por el profesor en la clase anterior, traslada el modo de representación geométrica al modo de representación numérica/algebraica y realiza los cálculos con ayuda de la construcción de este último modo [28-34].

\section{P4. El control de la estructura discursiva no implica el control del contenido}

Este episodio de interacción se inicia con una presentación con la que se pretende que los alumnos comprendan de manera general lo qué es «semejanza». Para tal fin, el profesor se apoya en tres láminas con figuras (un par de martillos, uno de árboles y uno de campanas, los cuales se diferencian entre sí por el tamaño).

Tabla 5. El control de la estructura discursiva no implica el control del contenido

\begin{tabular}{|l|l|}
\hline 01 & El maestro escribe en el centro del pizarrón la palabra "semejanza" (Murmullo de voces). \\
02 & Maestro: ¡Shhh! Silencio, por favor jóvenes. Utilizamos la palabra "semejanza", cuando observamos que \\
03 & algunos objetos, cosas o personas, tienen cierto parecido o comparten algunas características, ¿si se dan \\
04 & cuenta? \\
05 & Alumnos: ¡Sí! (en coro). \\
06 & Maestro: A continuación, les voy a dar un contenido para que lo tengan y podamos analizarlo. En su \\
07 & cuaderno anoten lo siguiente: \\
08 & Alumno: ¡Espéreme! \\
09 & Maestro: «Con frecuencia utilizamos la palabra semejanza...». \\
10 & Alumno: ¡Espéreme! \\
11 & Maestro: «... para decir que dos figuras, cosas, objetos o personas, son parecidos en algunos aspectos...». \\
12 & Alumno: ¿Objeto qué? \\
13 & Maestro: Y bueno, ya que tenemos esa definición, les daré algunos ejemplos que les ayudarán para \\
14 & comprender lo que de manera general es la semejanza. \\
15 & \\
16 & \\
17 & \\
18 &
\end{tabular}

El maestro inicia la clase escribiendo en la parte central del pizarrón la palabra «semejanza», lo que provoca murmullos en el salón de clases, así es que los alumnos no están atendiendo a las acciones del profesor. Él intenta poner orden en la clase ([02] "Shhh! Silencio, por favor jóvenes") y al no encontrar respuestas de los alumnos continúa hablando "Utilizamos la palabra semejanza, cuando observamos que..."). Hasta este momento, el profesor no tiene el control de la clase, se observa un poco nervioso, dirige la mirada hacia los lados. La clase es interrumpida por alumnos que llegan tarde; entonces de nueva cuenta aparece el descontrol en el grupo, mientras que el profesor pregunta ([03-04] “isi se dan cuenta?") y los alumnos responden en coro ([05] "isí!"). El profesor intenta mantener el control de la clase con la introducción del nuevo contenido temático ([06-07] "A continuación, les voy a dar un contenido para que lo tengan y podamos analizarlo. En su cuaderno anoten lo siguiente..."), los alumnos retoman el ritmo de la clase con interrupciones como: [08] “iEspéreme!”, [13] “¿Objeto qué?”). Después de escribir la definición que les dio el profesor, continúan con sus respectivos diálogos; situación que provoca un ruido excesivo en tanto el profesor, auxiliado por un alumno, coloca tres láminas con figuras (ver tabla 5, líneas 16-23). 
En este breve análisis del episodio de la tabla 5, ya se ha preparado el terreno para un análisis más puntual de cómo alumnos y profesor intentan controlar el comportamiento mutuo, además de la dirección de la clase. Alumnos y profesor tienen intereses diferentes y, a menudo, conflictivos en cuanto a lo que sucede en el aula. No es accidental que las invitaciones a iniciar casi siempre provienen del profesor y que los alumnos ponen cierta resistencia a dar inicio (platican con sus compañeros, sacan útiles, arrastran sillas, llegan tarde a clase, interrumpen al profesor, etc.). Al final de la clase son los alumnos quienes invitan acabar con la actividad (señalan la hora de salida, cierran cuadernos, inician el dialogo con algún compañero, etc.); los profesores ratifican estas invitaciones o negocian la disolución de la clase atendiendo a un timbre o a la hora oficial que determina el fin de la sesión.

\section{P5. La validación del contenido en la clase de geometría}

En virtud de la asimetría propia de la relación didáctica que vincula a quien domina un saber con quién debe aprenderlo, el profesor es el responsable de la validez generada en clase, como afirma Margolinas (1992) la fase de validación se refiere al momente en que se trata de saber si el resultado obtenido es el adecuado conforme al problema planteado. Esto significa que en la fase dedicada a la valoración de resultados de la actividad, el docente tiene que emitir un juicio directo sobre la validez del trabajo de los estudiantes. Es decir, es una actividad que propiamente le compete al profesor. En cambio, mediante la validación se otorga a los alumnos el lugar de sujetos epistémicos que pueden participar, sobre la base de la exposición de pruebas, en la decisión sobre la validez de los resultados obtenidos de la actividad. Como se muestra en episodio de la tabla 6 , es muy frecuente que el profesor demande que los alumnos argumenten sus explicaciones:

Tabla 6. La validación del contenido en la clase de geometría.

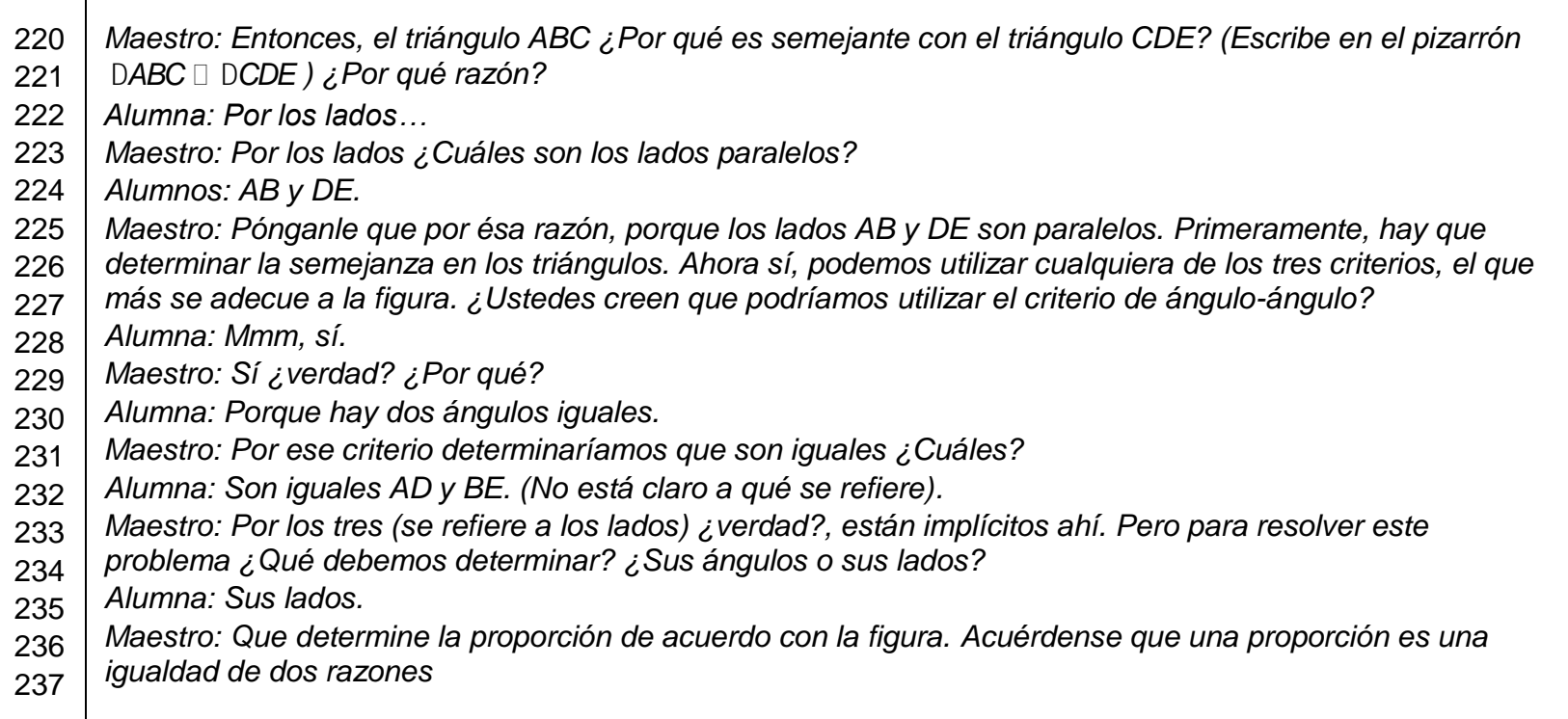

En el episodio de la tabla 6, el profesor planteó la siguiente pregunta: [220] "¿Por qué es semejante con el triángulo $C D E$ ?", posteriormente intentó involucrar a los alumnos en la construcción de pruebas a través del cuestionamiento: [221] “¿Por qué razón?”. Éste patrón que pretende construir una versión conjunta y compartida del conocimiento con sus estudiantes es usual en esta zona. Al parecer y en el principio así ocurre [225-237], pero los alumnos no ofrecen argumentos útiles que validen la proposición inicial ni aún a nivel empírico, el cual consiste en describir el procedimiento que condujo al resultado. Se observa también que el maestro decidió recuperar la responsabilidad didáctica que había transferido a los estudiantes al darse cuenta de que no emergían argumentos sólidos que conllevaran a demostrar por qué los dos triángulos son semejantes [236-237].

La zona de validación se establece a partir de los interrogatorios a los alumnos. Entonces, las contribuciones de los alumnos son incorporadas como contenidos del debate y, con frecuencia, se producen situaciones de habla al unísono. Tanto los alumnos como el maestro interrogan y contestan; el profesor conoce de antemano las respuestas, pero no se espera de él una retroalimentación evaluativa. El establecimiento de esta zona es el resultado de la participación conjunta que es desplegada mediante dos estrategias: construir una versión conjunta y compartida de conocimiento y la argumentación. La tabla 7 resume los patrones de interacción identificados en el discurso de los profesores con sus respectivas estrategias de enseñanza: 
Tabla 7. Patrones de interacción identificados en el discurso de los profesores

P1. Los contenidos primero se explican y después se aplican a ejercicios concretos.

1. El profesor plantea ejercicios para reafirmar lo explicado en clase.

2. El profesor contribuye a la solución del problema mediante preguntas adicionales, observaciones, reformulaciones o juicios, con una mínima actividad intelectual de parte de los alumnos.

3. El profesor le da más importancia a la parte procedimental que a lo conceptual.

P2. La explicación: una estrategia discursiva en la clase.

1. La explicación se establece bajo el despliegue de las siguientes estrategias: exposición oral e interrogatorio a los alumnos.

2. El profesor reformula las explicaciones de los alumnos.

3. El profesor provee explicaciones a través de un ejemplo

P3. Para que los conocimientos sean válidos, deben demostrarse.

1. El profesor demanda argumentaciones y justificaciones.

2. El profesor demanda explicaciones de parte del alumno.

3. El profesor solicita demostraciones.

P4. El control de la estructura discursiva no implica necesariamente el control del contenido.

1. El profesor mantiene el control de la clase con la introducción de un nuevo contenido.

2. El profesor formula preguntas abiertas o anticipatorias.

3. El profesor controla el ritmo de la clase con una mínima participación de los alumnos.

P5. La validación del contenido en la clase de geometría.

1. El profesor establece la validación a partir de los interrogantes a los alumnos.

2. El profesor utiliza el consenso como una forma de validación.

3. El profesor intenta construir una versión conjunta y compartida de conocimiento y la argumentación.

\section{DISCUSION FINAL}

Los resultados de nuestra investigación confirman que por medio del discurso se facilita la identificación de patrones de interacción entre el profesor y sus estudiantes. Los patrones identificados se caracterizan por la constitución de regularidades, que se estabilizan en el proceso de negociación de significados matemáticos. La asimetría en los roles del profesor y alumnos parece estar definida por la misma naturaleza de la tarea educativa y por el hecho de que el maestro es quien tiene el control de la clase. Dicho control con frecuencia se da en un contexto interactivo llamado por Lemke (1997) asimétrico. Los profesores se comportan asimétricos, cuando interrogan a los alumnos. Las respuestas de los alumnos están relacionadas a la totalidad de la pregunta del profesor. Sin embargo, los resultados revelan una importante presencia de las intervenciones de los alumnos, además de su influencia en la dinámica en el discurso que se genera en el aula, puesto que con sus preguntas y sus respuestas, en ocasiones, ponen en duda las aseveraciones de los maestros.

Escudero (2005) determina tres aproximaciones distintas a la "semejanza" como objeto de enseñanza: una que le denomina relación intrafigural, y que corresponde a la ausencia de la idea de transformar una figura en otra; como transformación geométrica vista como una herramienta, y, finalmente, la transformación geométrica como objeto matemático. En los episodios analizados se aprecia una cierta influencia con la aproximación a la semejanza dentro la relación intrafigural en donde se encuentra ausente la idea de transformar una figura en otra, limitando la potencialidad como articulador de nociones matemáticas, como la proporcionalidad y el Teorema de Thales.

En este trabajo, hemos podido identificar algunos patrones de interacción discursivos presente en las aulas de Enseñanza Media. Sin duda, el hecho de tratar un contenido específico en el aula condiciona en gran medida la aparición de ciertos patrones y no otros. Los resultados encontrados hacen evidente que las acciones como la demostración y la argumentación tienen poco peso en las clases de los profesores analizados en comparación con las acciones exposición oral, interrogatorio y explicación a través de ejemplos, que son con mucho, las que más se presentan en las clases observadas y que se articulan con lo explicativo.

Por último, la introducción de la perspectiva interaccionista y el análisis del discurso a la Matemática Educativa, puede visualizarse como un posible punto de arranque para otros estudios que intenten involucrarse en el discurso matemático escolar y en su relación con la actividad docente. Esta relación permitirá comprender, con profundidad, los diversos procesos existentes en la enseñanza de las matemáticas, mismos que se construyen y reconstruyen en la Enseñanza Media Universitaria. 


\section{CONCLUSIONES}

A partir del análisis y reflexiones de los resultados, podemos llegar a las siguientes conclusiones:

1. En general, los profesores hacen evidente que las acciones como la demostración y la argumentación tienen poco peso en las clases, en comparación con las acciones de exposición oral, interrogatorio y explicación a través de ejemplos.

2. La explicación es una estrategia discursiva en la clase, bajo el despliegue de las siguientes estrategias: exposición oral del maestro, interrogatorio a los alumnos y explicación a través de un ejemplo.

3. La validación del contenido se establece a partir de interrogatorios a los alumnos y es desplegada a través de dos estrategias: construir una versión conjunta y compartida de conocimiento y la argumentación.

4. Los profesores organizan el contenido asociado al concepto de "semejanza" desde una cierta influencia dentro la relación intrafigural, en donde se encuentra ausente la idea de transformar una figura en otra, limitando la potencialidad como articulador de nociones matemáticas, como la proporcionalidad y el Teorema de Thales.

5. Nuestra contribución, dentro del ámbito metodológico, estriba en articular el método etnográfico con la perspectiva interaccionista y análisis del discurso, para la interpretación de procesos de interacción en el aula de Enseñanza Media Universitaria.

\section{REFERENCIAS}

Balacheff, N., Preuve est demonstration en mathématiques au collège, Recherches en Didactique des Mathématiques, 3(3), 261-304 (1982)

Barboza, J. A. y H. A. Zapata, El Estudio de clase, Estrategia y Escenario para la Cualificación del Profesor de Matemáticas, Formación Universitaria, 6(4), 49-62, (2013)

Bauersfeld, H., Language games in mathematics classroom: Their function and their effects. En P. Cobb y $\mathrm{H}$. Bauersfeld (Eds.), Emergence of mathematical meaning: Interaction in classroom cultures, Lawrence Erlbaum Associates, pp. 271-292, New Jersey, Estados Unidos, (1995)

Bertely, M., Conociendo nuestras escuelas. Un acercamiento etnográfico a la cultura escolar, Volumen 6. Paidos, México DF, México, (2010)

Brousseau, G., N. Brousseau y V. Warfiel, Teaching fractions through situations: A fundamental experiment Springer, New York, Estados Unidos, (2014)

Candela, A. Ciencia en el aula: los alumnos entre la argumentación y el consenso, Paidós, Barcelona, España, (1999)

Chevallard, Y., Estructures and fonctions. En J. L. Dorier y otros cuatro autores (Eds.), Actes de la 11e Ecole d' été de didactique des mathématiques, La pensée Sauvage, pp. 3-22, Grenoble, France, (2002)

Cobb, P. y H. Bauersfeld, Introduction: The coordination of psychological and sociological perspectives in mathematics education. En P. Cobb y H. Bauersfeld (Eds.), Emergence of mathematical meaning: Interaction in classroom cultures, Lawrence Erlbaum Associates, pp. 1-16, New Jersey, Estados Unidos, (1995)

Contreras, S. A., Pensamiento Pedagógico en la Enseñanza de las Ciencias. Análisis de las Creencias Currículares y sus Implicancias para la Formación de Profesores de Enseñanza Media, Formación Universitaria, 9(1), 15-24, (2016)

Erickson, F., Métodos cualitativos en investigación sobre la enseñanza. En M. Wittrock (Ed.), La investigación de la enseñanza II, Paidós Educador, pp. 195-301, México DF, México, (1986)

Escudero, I., Un análisis del tratamiento de la semejanza en los documentos oficiales y textos escolares de matemáticas en la segunda mitad del siglo XX. Enseñanza de las Ciencias, 23(3), 379-392 (2005)

Gavilán, J., M. García y S. Llinares, Una perspectiva para el análisis de la práctica del profesor de matemáticas, Implicaciones metodológicas, Enseñanza de las ciencias, 25(2), 157-170 (2007) 
Geertz, C., Interpretación de las culturas, Editorial Gedisa, México DF, México, (1987)

Godino, J., Categorías de análisis de los conocimientos del profesor de matemáticas. Revista Iberoamericana de Educación Matemática, 20, 13-31 (2009)

Gresalfi, M. y P. Cobb, Negotiating a vision of high-quality mathematics teaching in the context of professional development. Journal for Research in Mathematics Education, 42, 270-304 (2011)

Herbst, P., T. Nachlieli y D. Chazan, Studying the Practical Rationality of Mathematics Teaching: What Goes Into "Installing" a Theorem in Geometry? Cognition and Instruction, 29(2), 218-255 (2011)

Knippling, C., A method for revealing structures of argumentations in classroom proving processes. Mathematics Education, 40, 427-441(2008)

Krummheuer, G., Representation of the notion "learning-as-participation" in everyday situations of mathematics classes, Zentralblatt für Didaktik der Mathematik (ZDM), 43(12), 81-90 (2011)

Lemke, J. L., Aprender a hablar ciencia. Lenguaje, aprendizaje y valores, Paidós Ibérica, España, (1997)

Margolinas, C., Éléments pour l' analyse du rôle du maître: les phases de conclusión. Recherches en Didactique des Mathématiques, 12(1), 113-158 (1992)

Mercer, N., Palabras y mentes. Cómo usamos el lenguaje para pensar juntos, P aidós lbérica, España, (2001).

Perrin Glorian, M. y M. Hersant, Milieu et contrat didactique, outils pour l'analyse de séquences ordinaires, Recherches en Didactique des Mathématiques, 23(2), 217-276 (2003)

Pimm, D., El lenguaje matemático en el aula, Ediciones Morata, España, (1999)

Planas, N., One speaker, two lenguages: learning opportunities in the mathematics classroom, Educational Studies in Mathematics, 87(1), 51-66 (2014)

Ponte, J. y O. Chapman, Prospective mathematics teachers' learning and knowledge for teaching. En L. D. English y D. Kirshner (Eds.), Handbook of International Research in Mathematics Education, 3a Edición, Springer, pp. 275-296, New York, Estados Unidos, (2016)

Sfard, A., When the rules of discourse change, but nobody tells you: making sense of mathematics learning from a commognitive standpoint, Journal of the Learning Sciences, 16(4), 565-613 (2007)

Sierpinska, A. y S. Lerman, Epistemologies of mathematics and of mathematics education. En A. Bishop y otros cuatro autores(Eds.), International Handbook of Mathematics Education, Kluwer Academic Publisher, pp. 827-876, Dordrecht, The Netherlands, (1996)

Voigt, J., Negotiation of mathematical meaning and learning mathematics, Educational Studies in Mathematics, 26, 275-298 (1995) 